Voix et Images

\title{
Les essais québécois contemporains au confluent des discours
}

\section{René Audet et Pascal Riendeau}

Volume 39, numéro 3 (117), printemps-été 2014

Les essais québécois contemporains au confluent des discours

URI : https://id.erudit.org/iderudit/1026209ar

DOI : https://doi.org/10.7202/1026209ar

Aller au sommaire du numéro

Éditeur(s)

Université du Québec à Montréal

ISSN

0318-9201 (imprimé)

1705-933X (numérique)

Découvrir la revue

Citer ce document

Audet, R. \& Riendeau, P. (2014). Les essais québécois contemporains au confluent des discours. Voix et Images, 39(3), 7-16.

https://doi.org/10.7202/1026209ar d'utilisation que vous pouvez consulter en ligne.

https://apropos.erudit.org/fr/usagers/politique-dutilisation/ 


\title{
LES ESSAIS QUÉBÉCOIS CONTEMPORAINS AU CONFLUENT DES DISCOURS
}

\author{
$+++$ \\ RENÉ AUDET \\ Université Laval \\ PASCAL RIENDEAU \\ Université de Toronto
}

On n'associe pas spontanément la pratique de l'essai au Québec et la période actuelle. Le genre n'y possède pas une très grande visibilité ni ne fait l'objet d'une valorisation particulière. Présent mais diffus, il semble s'être réfugié dans divers créneaux, prendre des teintes variées qui s'inspirent des pratiques discursives apparentées. Ces stratégies, ces déplacements, nous souhaitons en faire une esquisse à travers la dernière vingtaine d'années. Que doit-on penser de la production essayistique québécoise depuis 1990 ou 1995 ? Le premier piège, en ce qui concerne ce genre, relève de sa nature et de sa définition, qui restent toujours problématiques. Tout en misant principalement sur sa déclinaison littéraire, nous avons voulu conserver une vision plus large de l'essai, en refusant notamment de lui accoler un adjectif. Sa diversité est ce qui le rend difficile à cerner, mais sans doute aussi ce qui constitue sa force, sa pertinence. Il existe une nécessaire (ou inévitable) hybridité de l'essai. Les textes critiques qui ont contribué à façonner la théorie (à partir des propositions de Georg Lukács, en passant par celles de Theodor Adorno, jusqu'à aujourd'hui) ont tous insisté, de différentes manières, sur cette hybridité intrinsèque de l'essai, qui se manifeste par la dualité, soit entre la littérature et la philosophie, le réel et le fictif, le soi et le monde, l'individu et la culture. Que signifie donc se situer au confluent des discours? Les articles de ce dossier répondent (directement ou indirectement) à cette question en montrant comment l'essai, discours subjectif, analyse un état de la culture, mais aussi de quelles manières s'expriment le littéraire, la littérarité — ou alors, comment la littérature (comme point de vue, comme posture) s'affirme dans et par l'essai.

Dans un dossier récent de la revue Contre-jour, les collaborateurs ont accepté de réfléchir, assez librement, à la possible disparition de l'essai en s'arrêtant plus particulièrement à la situation québécoise. D'entrée de jeu, le responsable du dossier, Étienne Beaulieu, pose cette question angoissée: «Se pourrait-il que nous assistions aujourd'hui, pratiquement impuissants, à la disparition progressive de l'essai littéraire ${ }^{1}$ ?» Une telle interrogation n'est pas nouvelle. Dans un article souvent cité, "Petite essayistique ${ }^{2}$ ",

1 Étienne Beaulieu, «Liminaire», Contre-jour. Cahiers littéraires, n²5, automne 2011, p. 57. 2 André Belleau, "Petite essayistique», Surprendre les voix, Montréal, Boréal, 1986, p. 85-89. Cet article a d'abord paru dans la revue Liberté (vol. XXV, nº 6, 1983, p. 7-10). 
André Belleau émettait, il y a trente ans, l'hypothèse que l'existence même de l'essai dépendait directement de la capacité culturelle d'une société à engendrer des essayistes :

l'apparition d'essayistes dans la littérature suppose une condition supplémentaire: que la teneur en culture du discours social ne se situe pas au-dessous d'un certain seuil. Car l'essayiste, lui, travaille plus spécifiquement avec le langage de la culture. Et il m'apparaît évident qu'une société où les signes de la culture sont raréfiés produira peu d'essayistes. Il serait facile d'imaginer la culture comme un gaz rare dans une société saturée de discours sportifs, publicitaires, etc. ${ }^{3}$

Belleau n'était pas prophète, mais on lui donne néanmoins raison quant à la présence de certains discours qui pourraient déliter la place de la culture et plus particulièrement l'espace de réflexion propre à l'essayiste. Or l'essai semble avoir réussi - mais pas toujours sans difficulté - à s'adapter à la transformation de la culture ou plutôt à sa mouvance. L'essai, comme les autres genres littéraires, en viendrait à s'exprimer différemment, prendrait d'autres formes et occuperait d'autres lieux que ceux qui ont marqué son établissement au xx $x^{e}$ siècle. En ce sens, il est réjouissant de voir que divers types de paroles essayistiques se retrouvent au sein des médias numériques. Pensons notamment aux blogues de Marie-Christine Lemieux-Couture - ses «Essais de néantologie appliquée» - et de Mathieu Arsenault, «Doctorak, go!» ${ }^{4}$. Il nous est ainsi apparu nécessaire, à la lumière de cet état de fait et en complément de cette réflexion sur la possible disparition de l'essai au Québec proposée par Contre-jour, d'engager plusieurs collègues spécialistes de l'essai dans un examen de la production essayistique très récente ${ }^{5}$.

\section{THÉORIE ET CRITIQUE}

Les études littéraires québécoises ont beaucoup contribué à l'analyse et à la théorisation de l'essai. Depuis le début des années 1980, de nombreuses études et anthologies importantes ont été publiées - pensons à celles de Laurent Mailhot ${ }^{6}$, de Jean Marcel ${ }^{7}$,

3 André Belleau, «Petite essayistique», p. 88. 4 On peut lire le blogue de Lemieux-Couture à l'adresse suivante: http://voir.ca/marie-christine-lemieux-couture/; celui d'Arsenault, à cette adresse-ci: http:// doctorak-go.blogspot.ca/. 5 De façon à bonifier cette mise en place initiale de la réflexion sur l'essai au Québec, nous avons souhaité greffer quelques regards émanant d'acteurs de l'essai tel qu'il se pratique dans les revues. En plus de passages tirés de ce dossier de Contre-jour, nous joignons ponctuellement des commentaires recueillis par correspondance privée : Julien Lefort-Favreau a généreusement accepté de répondre à nos questions, à titre de directeur du cahier critique (littérature) de la revue Liberté (correspondance du 4 juillet 2013); Nicolas Langelier, directeur d'Atelier 10 et fondateur de la revue Nouveau Projet, a fait preuve de la même générosité envers nous (correspondance du 17 septembre 2013). Nous les remercions de leur collaboration. 6 Laurent Mailhot, Ouvrir le livre, Montréal, l'Hexagone, coll. «Essais littéraires», 1992, 351 p. ; L'essai québécois depuis 1845. Étude et anthologie, Montréal, Éditions HMH, coll. "Cahiers du Québec. Littérature», 2005, 357 p. 7 Jean Marcel, Pensées, passions et proses, Montréal, l'Hexagone, coll. "Essais littéraires », 1992, $399 \mathrm{p}$. 
de Robert Vigneault ${ }^{8}$, de François Dumont ${ }^{9}$, de Jean-François Chassay ${ }^{10}$. Marc Angenot avait déjà amorcé une réflexion approfondie sur la question avec La parole pamphlétaire ${ }^{11}$. Si Angenot accordait une place somme toute restreinte à l'essai proprement dit dans cet ouvrage majeur et s'il ne parlait pas des essais québécois, ses hypothèses se sont rapidement trouvées à l'origine d'une tradition théorique et critique sur l'essai au Québec ${ }^{12}$. C'est, bien entendu, sans oublier les quelques courts articles d'André Belleau écrits à la même époque, qui continuent d'alimenter le débat. La présence d'un tel corpus critique nous parait essentielle pour mieux comprendre la situation actuelle de l'essai québécois. L'identification de nouvelles figures d'essayistes au tournant du XXI siècle, qui succèdent aux auteurs plus marquants des décennies précédentes, nous conduit inévitablement à vouloir nous saisir des conceptions singulières de l'essai et du discours sur la culture qu'elles favorisent, voire promeuvent. De quelles façons les textes idéels jouent-ils un rôle dans la définition même de la littérature québécoise? Les essais publiés au Québec depuis 1995 font preuve d'une vitalité certaine et d'une diversité indéniable, même s'il est vrai que plusieurs de ces essais ont connu une diffusion limitée et une faible réception critique. Devient-il alors inévitable de parler de l'effacement de l'essai dans l'espace intellectuel et culturel? Devrait-on être nostalgique de l'époque (récente) où La petite noirceur ${ }^{13}$ de Jean Larose ou La génération lyrique ${ }^{14}$ de François Ricard étaient considérés, à leur parution, comme l'essai le plus important de l'année au Québec ${ }^{15}$ ?

\section{POÉTIQUES DE L'ESSAI : ADHÉRER OU NON À LA TRADITION}

Afin de mieux connaître le corpus essayistique québécois paru depuis une vingtaine d'années, il nous importait à la fois d'examiner la poétique spécifique de l'écriture de l'essai contemporain et de procéder à une lecture culturelle d'un corpus d'essais. Comment la culture québécoise (au sens le plus large du terme) a-t-elle été observée, pensée, disséquée, mais aussi construite par les essayistes d'aujourd'hui? Si les «géants» de la Révolution tranquille ont modelé la pensée au moment du passage

$$
++
$$

8 Robert Vigneault, L'écriture de l'essai, Montréal, l'Hexagone, coll. «Essais littéraires », 1994, 330 p. ; Dialogues sur l'essai et la culture, Québec, Presses de l'Université Laval, coll. «Cultures québécoises », 2008, 75 p. 9 François Dumont, Approches de l'essai. Anthologie, Québec, Nota bene, coll. «Visées critiques», 2003, 276 p. ; François Dumont (dir.), La pensée composée. Formes du recueil et constitution de l'essai québécois, Québec, Nota bene, coll. «Cahiers du Centre de recherche en littérature québécoise de l'Université Laval», 1999, 286 p. 10 Jean-François Chassay (dir.), Anthologie de l'essai au Québec depuis la Révolution tranquille, Montréal, Boréal, 2003, 271 p. 11 Marc Angenot, La parole pamphlétaire. Contribution à la typologie des discours modernes, Paris, Payot, coll. «Langages et sociétés », 1982, 425 p. 12 En plus des ouvrages déjà cités s'inscrivent dans cette tradition critique le collectif Parcours de l'essai québécois (1980-2000), dirigé par Anne Caumartin et Martine-Emmanuelle Lapointe (Québec, Nota bene, coll. "Essais critiques», 2004, 220 p.), et le dossier coordonné par René Audet intitulé «Dérives de l'essai» (Études littéraires, vol. XXXVII, n 1, automne 2005). 13 Jean Larose, La petite noirceur, Montréal, Boréal, coll. «Papiers collés», 1987, 203 p. 14 François Ricard, La génération lyrique. Essai sur la vie et l'œuvre des premiers-nés du baby-boom, Montréal, Boréal, 1992, 282 p. 15 En écho à cette question, Julien Lefort-Favreau affirme : «L'époque où Jean Larose pouvait passer à la télévision semble bel et bien révolue. Nulle nostalgie de ma part; Mathieu Arsenault n'aurait pas pu tenir un blogue à l'ère de La petite noirceur.» Extrait d'une correspondance privée avec Julien Lefort-Favreau. 
d'une culture canadienne-française à une culture québécoise et de l'accession à la modernité, quelle place occupent les essais publiés au tournant du xxi siècle? Comment prendre acte de ce que l'on pourrait appeler «de nouvelles formes d'engagement» ou de la notion plus souple de «responsabilité» des essayistes dans la définition de la culture, à la rencontre des discours sociaux qui réactivent à la dure les notions - auparavant perçues comme surannées - d'éthique et d'expérience du monde? De quelle manière ces expérimentations modulent-elles la poétique contemporaine de l'écriture de l'essai ? Celle-ci se saisit mieux lorsque l'on étudie attentivement la mobilisation des discours par les essayistes d'aujourd'hui. L'essai peut être conçu comme une forme d'écriture singulière, ouverte sur les idées, qui témoigne des changements ou des transformations de la culture québécoise et du rapport de celleci avec le monde.

Le positionnement des discours à l'égard de la culture ambiante et de l'opinion courante - y adhérer ou s'en détacher - est en partie tributaire de l'appartenance générationnelle des essayistes, et plus particulièrement de la présence, dans l'espace social, d'essayistes plus jeunes. Cet élément se retrouve en effet parmi les discours récurrents qui interrogent la vitalité et la pérennité de l'essai. Belleau en résumait efficacement l'enjeu:

\begin{abstract}
La formation d'un essayiste exige beaucoup plus de temps que celle d'un poète ou d'un romancier. Je le dis sans ironie. À dix-huit ans, on peut être Rimbaud, on ne peut pas être un essayiste. La raison en est simple [...] : l'essayiste travaille dans le champ culturel avec les signes de la culture. [...] Or la connaissance et la maîtrise des langages qui composent le monde culturel se révèlent une entreprise infiniment plus longue que la connaissance et la maîtrise des formes romanesques destinées à représenter les langages sociaux de l'existence ${ }^{16}$.
\end{abstract}

Dans la présentation de son anthologie, Jean-François Chassay déplorait le fait qu'il n'avait pas réussi à intégrer des textes d'essayistes québécois importants de moins de quarante ans. Cela, écrivait-il en 2003, «correspond à une réalité de l'essai québécois aujourd'hui ${ }^{17}$ ». Chassay n'avait sans doute pas tort, et aujourd'hui encore, peu d'auteurs dans la trentaine pourraient être qualifiés d'essayistes majeurs. En revanche, parmi les essais qui se signalent (ou qui se taillent une place enviable dans le discours public), on en trouve justement quelques-uns écrits par ceux que l'on peut (encore) considérer comme de jeunes auteurs: Samuel Archibald, Nicolas Langelier ou Nicolas Lévesque ${ }^{18}$. À quels facteurs doit-on attribuer la présence plus limitée des jeunes

$+++$

16 André Belleau, Surprendre les voix, p. 88. 17 Jean-François Chassay, «Introduction », Anthologie de l'essai au Québec depuis la Révolution tranquille, p. 9. 18 Voir Samuel Archibald, Le sel de la terre. Confessions d'un enfant de la classe moyenne, Montréal, Atelier 10, coll. «Documents», 2013, 87 p.; et Nicolas Langelier, Année rouge. Notes en vue d'un récit personnel de la contestation sociale au Québec en 2012, Montréal, Atelier 10, coll. «Documents», 2012, 100 p. En ce qui concerne Nicolas Lévesque, pensons surtout à [...] Teen spirit. Essai sur notre époque (Québec, Nota bene, coll. «Nouveaux essais Spirale», 2009, 147 p.) et, plus récemment, à son essai à quatre mains : Ce que dit l'écorce (avec Catherine Mavrikakis, Québec, Nota bene, coll. «Nouveaux essais Spirale», 2014, 169 p.). 
essayistes sur la scène éditoriale? D’après Nicolas Langelier, "pour beaucoup de jeunes gens brillants et cultivés, l'essai n'est plus la forme incontournable qu'elle était pour les générations précédentes. [...] Est-ce à dire que tout est perdu? Je suis un optimiste, et je crois qu'une véritable renaissance est possible. Mais il faut être conscient que celle-ci se fera dans un contexte de créneau restreint ${ }^{19}$ ». Les éditeurs laissent-ils suffisamment la place aux jeunes? Selon Julien Lefort-Favreau, certaines collections importantes d'essais, dont "Papiers collés » et "Liberté grande » au Boréal, " font très peu de place (voire pas du tout) aux plus jeunes». Il ajoute qu' «il semble difficile de voir se former une parole essayistique hors du pamphlet journalistique (Mathieu Bock-Côté) et de l'essai universitaire ${ }^{20} »$. Le rapport de l'essai avec les déterminants sociaux et culturels semble, dans les circonstances, soumis à différents réajustements.

\section{LES ESSAYISTES}

Durant la période étudiée, de nombreuses œuvres d'essayistes aguerris - qui inscrivent leur réflexion dans une démarche plus spécifiquement littéraire - se sont poursuivies (François Ricard, Jean Larose, Jacques Brault, Pierre Vadeboncœur). De nouvelles voix sont venues s'ajouter, que ce soit celles d'Yvon Rivard, de François Paré ou encore de Régine Robin, dont l'œuvre considérable (en fiction narrative, en études littéraires) s'est enrichie de textes plus personnels qui n'excluent pas les accents polémiques. L'œuvre essayistique lucide et sombre de René Lapierre a vraiment commencé au début des années 1990, et s'est établie comme l'une des plus importantes et des plus fortes. Quant à Pierre Nepveu, il serait tentant d'en faire un cas à part, puisqu'il «passe de la critique savante à l'essai littéraire sans rupture, par l'amplification d'un certain nombre de traits formels (digressions, rêveries sur les textes, inscription de plus en plus marquée du sujet) ${ }^{21}$ ». Nombreux sont aussi les romanciers et dramaturges qui pratiquent l'essai. Certains le font de manière plus occasionnelle (Marie-Claire Blais, Normand Chaurette, Dany Laferrière, Monique LaRue, Pierre Samson ou le cinéaste Bernard Émond), d'autres passent plutôt par la chronique (Louis Hamelin, Nicolas Dickner). Quelques autres ont une pratique plus soutenue (Pierre Morency, Suzanne Jacob, Roland Bourneuf). Quant aux voix émergentes comme celles de Nicolas Lévesque, de Nicolas Langelier ou de Samuel Archibald, elles donnent peutêtre déjà une idée d'une tendance de l'essai aujourd'hui, où l'extrême actualité sert de déclencheur et de contexte à une parole en procès. Cette énumération d'essayistes, incluant les auteurs qui n’ont écrit qu'un essai, montre que malgré des difficultés évidentes ou un contexte culturel défavorable, ce genre s'est trouvé à réaffirmer sa pertinence au sein de la littérature québécoise.

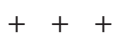

19 Extrait d'une correspondance privée avec Nicolas Langelier. 20 Extrait d'une correspondance privée avec Julien Lefort-Favreau. 21 Michel Biron, François Dumont et Élisabeth Nardout-Lafarge, avec la collaboration de Martine-Emmanuelle Lapointe, Histoire de la littérature québécoise, Montréal, Boréal, 2007, p. 602. 
Les lieux de publication deviennent aussi des éléments déterminants pour permettre le développement d'une pluralité de paroles essayistiques. Si un nombre limité d'éditeurs font leur part - pensons au Boréal, aux Herbes rouges, à Nota bene ${ }^{22}-$, l'essai naît souvent dans les revues. Liberté, L'Inconvénient, Contre-jour et Nouveau Projet peuvent sans doute être considérées comme les quatre principales revues produites en 2014 au Québec où l'essai s'exprime le plus librement. De manières différentes et à divers degrés, ces revues accueillent des paroles d'essayistes originales et misent sur l'importance d'une écriture littéraire ${ }^{23}$, phénomène qui s'est bien implanté d'abord dans la revue Liberté, «véritable pépinière des essayistes de la décennie 1980, dont le point commun est sans doute la défense de la littérature, moins comme ensemble patrimonial de textes que comme mode de connaissance (de soi et du monde) et d'intervention ${ }^{24}$ ». La longévité de Liberté et la capacité qu'elle a eue à se réinventer la placent dans une catégorie à part, où est maintenant privilégiée « une voie intermédiaire entre l'université et le journalisme ${ }^{25}$ ", affirme Lefort-Favreau. «D'autres revues reprendront le flambeau de la littérature comme valeur dans les années 2000, poursuitil dans son commentaire sur le rôle actuel des revues dans le développement de l'essai au Québec, L'Inconvénient [...], où c'est l'ironie qui définit d'abord la littérature, et Contre-jour [...], qui oppose à cette conception la dimension éthique de l'art ${ }^{26}$.» Dernière revue à apparaître dans le paysage éditorial québécois, Nouveau Projet s'est rapidement imposée dans le domaine de l'essai. Langelier explique dans quel contexte la revue a été créée:

[O]n a assisté - au cours de ces mêmes dix dernières années au cours desquelles le texte moyen a raccourci et s'est simplifié - à une certaine renaissance de l'essai. Par exemple, on a vu aux États-Unis la naissance de plusieurs petites revues qui misent sur l'essai [...]. Je pense en particulier à des revues comme $n+1$, Jacobin, The Point, etc. Celles-ci sont portées par des jeunes dans la vingtaine et la trentaine qui ont soif d'idées approfondies et de textes forts et bien écrits. On sent chez eux une certaine nostalgie de l'âge d'or des années 1940-1960 et un désir de recréer une effervescence intellectuelle.

C'est un sentiment similaire qui m’a poussé à fonder Nouveau Projet en 2012. Je pense que dans la médiasphère actuelle, qui mise avant tout sur l'image et les idées en capsule, il y a de la place pour les essais et les réflexions approfondies. On s'entend, cette place est une niche, un créneau restreint - mais elle est bien là, et c'est une espèce de devoir intellectuel et social que de l'occuper et de lui permettre de survivre ${ }^{27}$.

$$
+++
$$

22 Il faut également mentionner la collection «Écrire» des Éditions Trois-Pistoles, dans laquelle plus d'une vingtaine de textes ont été publiés, dont ceux de Nicole Brossard et de Suzanne Jacob. 23 Ce trait distingue ces quatre revues d'autres lieux faisant la part belle à l'essai (Argument, À bâbord!), plutôt marqués par une vision plus sociologique ou idéologique du texte essayistique. 24 Michel Biron, François Dumont et Élisabeth NardoutLafarge, avec la collaboration de Martine-Emmanuelle Lapointe, Histoire de la littérature québécoise, p. 599. 25 Extrait d'une correspondance privée avec Julien Lefort-Favreau. 26 Ibid. 27 Extrait d'une correspondance privée avec Nicolas Langelier. 
Les revues tendent ainsi à suppléer à un marché du livre de plus en plus précaire et à permettre une éclosion spontanée de lieux de publication dont la ligne éditoriale est très affirmée. Bien que les travaux critiques sur l'essai aient souvent décrit les revues comme des supports généralistes constituant un premier moment dans la vie des textes, avant leur reprise en livre, on observe dans la période actuelle une autonomisation et une spécialisation de ces lieux : ici des revues qui développent des créneaux très précis, là des collections d'essais originaux, dont la longueur intermédiaire est favorable à une efficace argumentative plus grande.

\section{LES ARTICLES}

Si les essais contemporains tendent à être des creusets d'interaction entre les discours, il ne s'agit toutefois pas pour les essayistes de chercher volontairement à effacer les frontières des disciplines ou des sphères sociales. Leur action se situe ailleurs, et surtout elle prend forme autrement. Recourant à la force heuristique du genre, à la démarche processuelle de l'essai (cette réflexion en procès), ils travaillent plutôt à rassembler les conditions nécessaires à une avancée des idées, à un positionnement un peu plus satisfaisant des pôles en présence - observations, valeurs, faits, arguments - à la façon des pièces d'un jeu d'échecs, comme autant de figurines d'une représentation miniature d'une guerre célèbre... sauf qu'on ne connaît pas à l'avance l'issue de la bataille.

C'est dans un tel contexte qu'intervient la notion de responsabilité, qui traverse explicitement ou implicitement l'ensemble des contributions de ce dossier. Elle est convoquée frontalement par Jean-François Chassay, dans un article où il propose une lecture des trois premiers «Documents» publiés dans le sillage de la revue Nouveau Projet. Les propositions essayistiques, voire romanesques de la période contemporaine ont souvent été décriées en raison de leur refus de l'engagement, de leur fuite par une ironie typiquement postmoderne où les positions contradictoires et les doubles négations finissent par faire s'étioler toute prise de position. À leur façon (bien singulière), mais dans un effet de résonance étonnant, La juste part ${ }^{28}$ de David Robichaud et Patrick Turmel, Année rouge de Nicolas Langelier et Le sel de la terre de Samuel Archibald tentent une échappée hors du postmoderne en refusant le détachement ironique, car «le présent compte», soutiennent-ils dans une volonté d'adhérer à une nouvelle forme d'authenticité. Peut-on parler de la voix d'une génération? C'est ce que propose Anne Caumartin dans son étude, en montrant que Nouveau Projet et les essais publiés par Atelier 10 expriment une «conscience éveillée par les mouvements d'indignation ${ }^{29}$ ", et que leur parole permet de repenser le rapport au réel et au social à travers l'expérience du monde.

Cette responsabilité est autrement palpable dans les écrits d'Yvon Rivard, qui appelle à une plongée de l'intellectuel dans l'expérience du monde, plongée propre à

28 David Robichaud et Patrick Turmel, La juste part. Repenser les inégalités, la richesse et la fabrication des grillepains, Montréal, Atelier 10, coll. «Documents», 2012, 97 p. 29 Anne Caumartin, "La pensée qui fourche. Dislocation de l'essai québécois contemporain", dans le présent dossier, p. 88. 
favoriser, souligne Sylvano Santini, une « action intelligente ${ }^{30}$ », une pensée à l'écoute de l'autre. L'inscription de l'essayiste dans la société devient un signal (ou dans certains cas un symptôme) d'une dialectique entre l'écrivain, la littérature et la société : la circonstance de l'enseignement est très présente chez Rivard, en écho notamment à cette figure du «maître ignorant» de Jacques Rancière. Telle inscription dans le milieu prend la forme, dans les chroniques de Nicolas Dickner parues dans Voir, d'une négociation lente et récursive de l'image sociale problématique de l'écrivain. Dans l'examen qu'elle propose de cette série de textes, Élisabeth Nardout-Lafarge montre bien que ce «Hors champ», lieu excentré mais zone de créativité, permet à Dickner de travailler peu à peu à " performer, dans sa propre écriture, la singularité d'une vision littéraire sur le monde ${ }^{31}$ ». La profession d'écrivain ne se présente pas comme une figure médiatique chez Dickner, bien au contraire : dans un effort de démystification, il la recadre dans son mandat d'interprétation du réel - à l'instar du roman, la chronique que Dickner pratique se définit comme «activité généraliste», aux frontières de l'artisanat et de l'enthousiasme encyclopédique du geek.

Par ce portrait émerge l'empreinte de la littérature dans la société, ou plutôt la littérature comme empreinte: elle est modalité de saisie d'un état du social, façon de témoigner de fractures, de déplacements, de nouveaux schèmes de perception. François Paré mobilise des œuvres (Patrice Desbiens, Herménégilde Chiasson) et des figures iconiques (Ida Papineau) de la Franco-Amérique de façon à questionner dans ses ouvrages, Les littératures de l'exiguité et Le fantasme d'Escanaba ${ }^{32}$, la possibilité même que puisse exister cette idée de communitas, et, de là plus fondamentalement, les modèles diasporaux. Benoit Doyon-Gosselin et Rachel Veilleux dessinent le parcours qui sépare et réconcilie les deux essais de Paré, jalons d'une réflexion sur la culture d'origine à travers l'examen de l'itinérance identitaire propre aux francophonies hors Québec.

Partant de son expérience d'immigration et de sa propre pratique littéraire, l'essayiste Régine Robin s'engagerait autrement, mais de façon complémentaire, dans le questionnement de cette identité québécoise, engagement dont témoignerait son récent opus Nous autres, les autres ${ }^{33}$. Le mouvement est celui, inévitable, d'une généralisation d'une situation singulière, la sienne propre, en un exposé de situation à l'intérieur duquel l'essayiste parle pour les autres, et donc à leur place, en conclut Joëlle Papillon. La position de Robin à l'égard de cette typologie des «nous» du discours identitaire québécois est symptomatiquement celle d'une marginalité - celle de l'observatrice qui tente de se placer hors de son objet, celle de l'immigrante dans son désir d'intégration et celle de l'intellectuelle - reconduisant le topos de l'écrivain incompris. Si l'essai constitue certes un lieu privilégié pour réfléchir aux enjeux de la culture et de l'identité, il est tout autant le terreau d'une ambiguité énonciative qui les empêche d'être totalement objectivés et qui plonge l'essayiste au cœur de la polémique.

$++$

30 Sylvano Santini, «L'action intelligente d'Yvon Rivard. De l'effacement de soi à l'expérience du pauvre», dans le présent dossier, p. 32. 31 Élisabeth Nardout-Lafarge, «"L'écrivain en habit de travail". La chronique "Hors champ" de Nicolas Dickner à Voir (2006-2012)", dans le présent dossier, p. 51. 32 François Paré, Les littératures de l'exiguité, Hearst, Le Nordir, coll. «Essais», 1992, 175 p. ; Le fantasme d'Escanaba, Québec, Nota bene, 2007, 183 p. 33 Régine Robin, Nous autres, les autres. Difficile pluralisme, Montréal, Boréal, coll. «Liberté grande», $2011,346 \mathrm{p}$. 
Émerge de façon singulière de ces six études la négociation constante de la distance que peut, que doit conserver l'essai par rapport à ses objets. On l'observe d'emblée dans la gestion de la proximité avec le social, avec l'actualité. Les trois ouvrages de la collection «Documents» étudiés par Jean-François Chassay se cristallisent justement autour d'une actualité récente, voire brûlante ; la tension entière de l'analyse du «nous » québécois discuté par Régine Robin tient à une incapacité de faire abstraction de son expérience personnelle pour arriver à donner la parole à un «nous» plus diversifié. Dans Personne n'est une île et Une idée simple ${ }^{34}$, la pratique de l'essai chez Yvon Rivard relève d'une tension entre le «moi » et le monde, en écho à la notion d'«inframince» de Marcel Duchamp, tension qui se résout par un mouvement de désubjectivation et d'anonymat. Recadrant les essais de Rivard à la lumière d'un héritage de la pauvreté (concurrent ici de la conception proposée par Jean Larose), Sylvano Santini montre le pouvoir expressif des figures du pauvre, de l'innocent et de l'ignorant qui sont à même de garder l'individu à distance pour plutôt favoriser l'action intelligente fondée sur l'idée de communauté - ici d'une façon idéale, alors que Dickner exprimait pareille préoccupation dans le contexte très concret de la collectivité.

Anne Caumartin fonde son étude sur cette distance problématique que l'essai tend à conserver avec l'actualité, autant que sur sa paradoxale proximité avec l'actualité tout en cherchant à s'en défaire. Cette actualité, souvent problématique pour l'essayiste - qui a besoin de temps, d'espace et de recul pour mieux fonder sa réflexion -, suscite toutefois la question de son engagement. Les différentes formes d'engagement n'ont certes pas disparu, mais elles restent plus difficiles à imposer à une époque où, à tort ou à raison, on tend à s'en méfier. C'est sans doute pourquoi le concept souple de responsabilité paraît plus apte à dire le rapport de l'essayiste au monde. Appeler à un sens de la responsabilité, c'est ce que Marie-Claire Blais et Yvon Rivard mettent en avant dans l'essai que chacun a publié dans la collection «Liberté grande » au Boréal ${ }^{35}$. La première privilégie le poétique dans sa convocation de l'histoire et des mouvements de contestation du passé récent, alors que le second explore comment il a cherché à fonder un rapport pédagogique profond et respectueux. Dans la même voie, la notion de responsabilité fonde l'œuvre cinématographique de Bernard Émond, laquelle doit être abordée conjointement avec ses essais, de ceux-ci comme de celle-là se dégageant, signale Caumartin, une même voix, qui met surtout l'accent sur l'ouverture vers l'Autre et convoque une notion essentielle: l'espoir.

S'impose à l'évidence une confluence de pratiques discursives au sein même de l'essai, lequel se trouve à participer tant de la littérature que du discours social. Il mobilise un vaste spectre de discours allant de l'argumentatif jusqu'à la fiction narrative, empruntant tout aussi bien les voies de l'autobiographie. La plongée dans des capsules d'inspiration autobiographique nourrit le propos tant chez Nicolas Langelier

34 Yvon Rivard, Personne n'est une île, Montréal, Boréal, coll. «Papiers collés», 2006, 258 p. ; Une idée simple, Montréal, Boréal, coll. «Papiers collés», 2010, 241 p. 35 Voir Marie-Claire Blais, Passages américains, Montréal, Boréal, coll. «Liberté grande», 2012, 96 p. ; et Yvon Rivard, Aimer, enseigner, Montréal, Boréal, coll. "Liberté grande», 2012, 202 p. 
que chez Samuel Archibald, avec là les doutes qui obsèdent l'écrivain, ici les mises en scène de soi qui ouvrent sur la fictionnalisation au fondement de son recueil d'histoires Arvida ${ }^{36}$. Yvon Rivard ne fait pas autrement quand il crée une résonance immédiate avec son personnage d'Alexandre, protagoniste du roman Le siècle de Jeanne ${ }^{37}$, refusant l'étanchéité entre les deux genres. La perméabilité des frontières s'observe dans l'œuvre de Bernard Émond, où la voix essayistique porteuse d'un appel à la responsabilité se manifeste autant dans les textes qu'à l'écran. Cette compénétration du discours et de l'image, de l'essai et de la fiction provoque ici une certaine mise à distance du soi, alors que d'autres essais refusent celle-ci entièrement. C'est ce qui s'observe chez Régine Robin, dont les écrits mobilisent constamment l'expérience singulière de l'universitaire, colorant (voire biaisant) la lecture qu'elle propose de l'identité nationale. Pourtant, l'inscription même de l'essayiste dans un milieu, dans un bassin culturel, est une condition sine qua non du développement de sa pensée. Cette idée se dessinant initialement dans le plaidoyer d'Yvon Rivard pour une pensée qui «avance dans l'expérience ${ }^{38}$ ", les articles de ce dossier l'illustrent de diverses façons, et la nostalgie de l'origine de François Paré la confirme avec force : la lecture répétée du récit matrice du départ éveille en lui une infinie tristesse, mais un départ dont l'expérience profonde chez lui est le gage d'une saisie fine et engagée du tourment des diasporas québécoises. L'intime tissage du récit d'un soi agissant et de la réflexion active sur le monde définit profondément l'essai, et la mise en relief de cette composition caractérise plus intensément l'essai québécois tel qu'il s'exprime au tournant du XXI siècle.

36 Samuel Archibald, Arvida, Montréal, Le Quartanier, coll. «Polygraphe», 2011, 314 p. 37 Yvon Rivard, Le siècle de Jeanne, Montréal, Boréal, 2005, 398 p. Ce personnage est également le protagoniste des Silences du corbeau (Montréal, Boréal, 1986, 265 p.) et du Milieu du jour (Montréal, Boréal, 1995, 327 p.). 38 Sylvano Santini, «L'action intelligente d'Yvon Rivard. De l'effacement de soi à l'expérience du pauvre», dans le présent dossier, p. 37. 